\title{
An Experience on Mechatronics Teaching on Undergraduate Students by Means of The Skybot Platform: from Classroom to Robot Competition
}

\author{
P. Gil, S. T. Puente, F. A. Candelas, I. Perea, G.J. García, C.A. Jara, J.A. Corrales \\ Physics, Systems Engineering and Signal Theory Department \\ University of Alicante, Crta. San Vicente del Raspeig s/n, 03690, Alicante, Spain \\ \{pablo.gil,santiago.puente,francisco.candelas,ivan.perea,gjgg,carlos.jara,jcorrales\}@ua.es
}

\begin{abstract}
This paper analyzes the learning experiences and opinions from a group of undergraduate students in a course about Robotics. The contents of this course were taught as a set of seminars. In each seminar, the student learned interdisciplinary knowledge of computer science, control engineering, electronics and other fields related to Robotics. The aim of this course is that the students are able to design and implement their own and custom robotic solution for a series of tests planned by the teachers. These tests measure the behavior and mechatronic features of the students' robots. Finally, the students' robots are confronted with some competitions. In this paper, the low-cost robotic architecture used by the students, the contents of the course, the tests to compare the solutions of students and the opinion of them are amply discussed.
\end{abstract}

\section{Keywords: Educational Robot, Low-Cost Robot, Learning}

\section{Introduction}

Robotics is becoming an important subject in education not only for undergraduate students at universities but also for schools and high schools students [1]. More and more courses, based on the design and implementation of robots, are developed for educational purposes due to the following three main features:

- Interdisciplinary: The interdisciplinary of robotic courses involves the combination of knowledge from different fields (such as electronics, software engineering, artificial intelligence, etc.) in order to get a final common goal (e.g. the construction of a robot). This property helps students to understand that the concepts that they learn during their courses are not isolated but they can be combined and merged in order to solve complex problems.

- Constructivism: Robotic courses usually follow a constructivist paradigm which helps students construct their own knowledge through practical exercises. In other words, students can learn robotics while designing and developing their own robots. This active learning process not only enables the contextualization of robotic concepts in real situations but it also increases the motivation of students because they see how theoretical concepts can be applied for developing real devices which interact with the world.

- Collaboration: Robotic courses are usually organized in groups of students where several students work cooperatively in order to build their robots. This collaboration not only allows students to learn from each other but it also increases their group management skills. In fact, students also learn to divide the work into different tasks, assigning them to each group member depending on his expertise, coordinate their execution and finally integrating them into a common project.

These three features not only help students to increase their motivation in STEM (Science, Technology, Engineering and Math) subjects, but they also represent skills which are required by most engineers in their daily work. This fact is quite interesting for future undergraduate students, who will soon abandon the academic world in order to enter the work world. Thereby, universities and enterprises get closer, so that the skills acquired at the academic world can be then applied at work. 
Educational robotics usually applies two different activities in order to develop these skills: robot building/programming courses and robot competitions. Only one or both activities can be applied, depending on the organization of the robotics subject. Two types of robotic platforms can be considered to develop these two activities, jointly or separately: humanoid robot [2] and mobile robots [3]. The student can learn programming skills and acquire knowledge from many areas of robotics research such as: tasks planning, movement control, sensitized and navigation in an environment with either of the two types of robots.

Nevertheless, most educational approaches combine them sequentially: firstly, students build and program their own robots and secondly, they verify and correct their robots behavior in a competition with other robots. In fact, this organization directly matches the different stages of engineering: analysis of requirements, design and implementation (during the building process of the robot) and verification and redesign (during the competition).

Many different robotic kits have been designed in the last years in order to develop robotic courses. Two main groups can be identified: commercial kits and personalized kits. Between commercial kits, VEX [4], K-Team [5] and LEGO Mindstorms [6] are the most commonly used [7]. In fact, 90\% of most courses [1] are based on LEGO Mindstorms because of its flexibility and its programming tools [8]. Nevertheless, commercial kits are usually expensive and hide many design details to the students. Because of this fact, there are robotic courses which are based on the design and building of a robot from scratch so that students acquire deeper knowledge and skills in mechanical and electronic design. In addition, they usually are more expensive than commercial kits because their cost only depends on the cost of their components. Several examples can be found in literature of this kind of personalized robotic kits. For instance, [9] proposes the Onubot robot which is able to follow lines. In [10], an open platform (EmbedIT) is proposed where several hardware modules can be interconnected in order to build different kinds of robots (such as a quadrupedal running robot and a skating robot). A tricycle mobile robot (Trikebot) with a camera for following lines and object tracking (via color) is presented in [7].

As stated before, another main activity of educational robotics is robotic competition. The competitions enhance the teamwork and collaboration skills of students since they have to interact in a group in order to improve their robot performance [11]. Many different robotic competitions have been developed in the last years [12]. For instance, the AAAI (American Association for Artificial Intelligence) robot competition has been taking place since 1992 and combines three or four different categories (such as robot navigation, robotic manipulation, human-robot interaction, robot rescue, etc.) [13]. Similarly, the Robocup competition is one of the most important international robot competitions, which includes contests about soccer, robot rescue and dancing [14]. It was founded in 1997 and it gets together more than 500 teams from more than 40 different countries. Other examples of more specific robot competitions are Cybertech [15] (whose main event is a simulation of bullfighting with mobile robots) and CEAbot [16] (which include different tasks with humanoid robots).

This paper presents the experiences of the authors in the development of educational Robotics at the University of Alicante (Spain). In work previous, the authors have developed virtual environments to simulate and remote control of robots [17], interactive tools for robotics simulations [18] and they have evaluated this type of tools [19]. Nowadays, the authors have developed the two main activities described above: a robot building/programming course and a robot competition. During the robot course, the students build their own mobile robot, a modified Skybot robot [20]; these educational robot built and programmed by the students can be classified as a UCRs [21]. Afterwards, when they have programmed some essential robot behaviors, they can test the robustness of their algorithms by competing in a robot contest. This paper is organized in the following sections in order to explain the main advantages of this approach for educational Robotics: section 2 describes the architecture of the robot and the modified electronics which have been added in order to improve their sensory capabilities, section 3 describes the organization of the robot course and explains the robot, in section 4 is analyzed the opinion of the students about both activities and finally, the last section draws the main conclusions from the results of both activities. 


\section{Skybot's architecture}

The robot Skybot, marketed by the company IFARA technologies [20], is a simple, economic, educational, and open robotic platform. Students (secondary, under graduate or bachelor students) who have an interest in robotics can start it in a fast and easy way by means of this open platform. It's an open robot because levels of the mechanical structure, the hardware schematics and the source code of the programs are available without any cost. Thus, anyone can build the robot and program it with only some knowledge about programming.

\subsection{Control board}

From the first moment when the course, described in this paper, was prepared, it was clear that the brain of the robot which students had to use should be based on a microcontroller. Basically, a microcontroller is a complete computer embedded in only one chip (integrated circuit) which includes the necessary units to run programs and interact with external devices. A typical microcontroller chip includes the following units: microprocessor, program memory, data memory, timers and counters, input and output ports (analogical and digital), serial communication ports, clock generator and power control. In this way, an integrated circuit with low-power consumption is achieved and it is easily replaceable.

A second issue that was overlooked was the PCB (Printed Circuit Board) in order to wire the microcontroller with other components, such as sensors and motors. Although design and assemble a PCB with a microcontroller are not complicated, they require skills in electronics that are out of the course objectives. Thus, it was decided to use a PCB developed by third parties.

Finally, in addition to the PCB with the microcontroller, it is also necessary to have a programming environment that allows students to develop programs and load them on the processor. Usually, microcontroller manufacturers provide tools that allow programming their processors in low-level languages that are efficient but difficult to learn.

Several development environments for microcontroller designs were studied to select the most appropriate to the course considering the facts previously mentioned. Mainly we have sought environments that offer a versatile PCB already designed, in addition to a programming environment easy to learn and use, while both hardware and software have a free license for use, modification and distribution, like GPL (GNU General Public License), in order to facilitate not only the integration into the course but also the use by students. Most notable choices today are the following:

- $\quad$ SkyPIC [23]. This board is based on PIC 16F876A microcontroller. PICs (Peripheral Interface Controller) are a very popular family of microcontrollers which is developed by Microchip Technology, and characterized by low cost and simple low-energy architecture, usually based on 8 bits technology. With the available development tools and libraries, PICs can be easily programmed in C language. The software is offered as GPL, and the hardware with a GPL-like licence. In addition, SKYPIC can be matched with the Sky293 board (Fig. 1), which has the necessary interfaces to control small motors and miniaturize several sensors, and it is appropriated to control little robots.

- $\quad$ Arduino [24]. This is a very popular board based on the ATmega series of microcontrollers from Atmel, which uses the 8-bit AVR RISC (Reduced Instruction Set Computing) architecture. This board is characterized by its low cost, versatility, ease of programming and the huge development community that uses Arduino for a wide range of projects. The Arduino platform is also known for its easy programming environment that has a large library of functions and lets users program in $\mathrm{C}++$ language. Thus, it is easy to find all types of devices which can be connected to the Arduino, such as communications modules, displays, sound cards, compasses, etc. However, to develop a mobile robot with this board, without having to resort to multiple interface circuits, the design of a more specialized interface board is required. Software and hardware are distributed with GPL licence. 
- $\quad$ Netduino [25] is a powerful version of Arduido with uses a 32 bits microcontroller from Atmel which can be directly in C\# language by using the Microsoft's .NET platform [23]. This is more adequate for people that know this language, like students finishing Computer Science Engineering. Hardware and basic software tools are offered with Creative Commons and Apache licences. Microsoft also provides a free version of its programming environment Microsoft Visual C\# Express.

- $\quad$ IOIO for Android [26] is a board based on the 16 bits PIC 24Fxxx. This board permit to interact with Android devices. Whereas Android is a free and source code platform, user requires to know this platform to program the microcontroller.

- RaspBerry [27] is the newest embedded PC low-cost, which can be used with a Linux distribution for ARM processors. It has 6 GPIO, Ethernet, host USB controller, 2 I2C interfaces, SPI, and one USB peripheral port. It incorporates a GPU unit in the microcontroller and 256 MiB of RAM. RaspBerry works at 700Mhz, and it can reproduce 1080p video quality.

Table 1. Economic cost

\begin{tabular}{c|c}
\hline Component & Prices (€) \\
\hline SkyPIC+Sky293 & from 20(unmounted) to 90(mounted) \\
Arduino & from 15 to 60 (depending on the version) \\
Netduino & 25 \\
IOIO & 40 \\
RaspBerry & 25 \\
Skybot robot & from 175 \\
\hline
\end{tabular}

Among the above mentioned options, the SkyPIC was finally chosen for the course. Main reason is that a complete kit for mounting the Skybot robot, including SkyPIC and Sky293 boards, is available with a cost of $175 €$ (Table 1). For the other alternatives was necessary to design a new chassis or modify an existing robot, since no specific robots chassis are available for them. Other important reason is the fact that the PIC 16F876A is widely used in commercial applications due to its low cost and low power consumption, and therefore it is specially interesting that students learn to program it. Finally, it is also worth mentioning that this microcontroller is distributed in a typical 28 pin DIL (Dual In Line) packaging, which is easy to replace in case of fail.
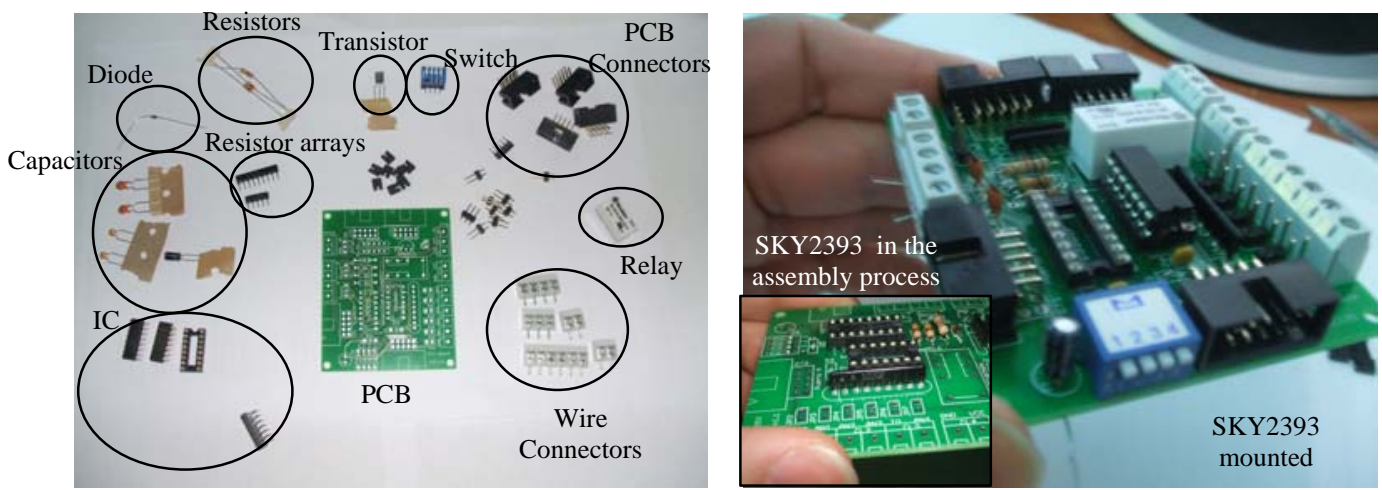

Figure 1. Components to assembly the SKY293 and SKY293 mounted.

More specifically, PIC 16F876A microcontroller includes the following units (Fig. 2): 8 bits processor, 368 bytes of RAM for data, 256 bytes of EEPROM (Electrically Erasable and Programmable ROM) for permanent data, 14KB of FLASH (Fast Erasable EEPROM) for storing the program, 2 communication serial ports (1 synchronous, 1 asynchronous), 3 timers-counters of 8 and 16 bits, 5 DCs (Analogical to Digital Converter) channels of 10 bits, and 2 CCP (Compare Counter and Pulse Width Modulation) outputs of 10 bits. These resources are sufficient to control a small robot as the Skybot.

With regard to the Sky293 board, this basically includes the interfaces for 2 little CC motors, 4 infrared detectors (which can be easily doubled), and five lines configurable as analogical inputs, 
digital inputs or digital outputs, which are commonly used to connect light and contact sensors such as LDR (Light Dependent Resistor) and bumpers push buttons.
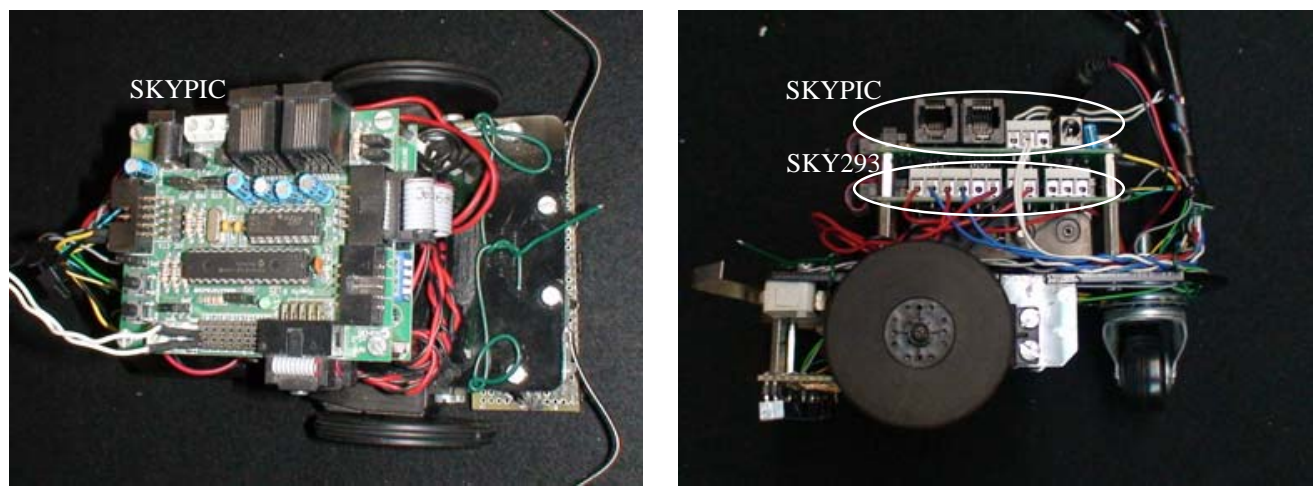

Figure 2. SKYPIC and SKY293 fitted one over the other.

\subsection{Chassis, motors and sensors}

The mechanical structure is composed of 7 pieces of plexiglass of $3 \mathrm{~mm}$, two 3003 Futaba servo motors and a caster wheel (Fig. 3a). It is an easily replicable structure and can be used materials such as wood, expanded PVC, aluminum, etc. The pieces are joined by glue and engines are attached by means of normal metric 4 screws. Both the screws and the caster wheel can be found at any hardware store. With regard to the servo-motors (Fig. 3b), they are standard servo motors of three pole of ferrite, controlled by PWM (Pulse-Width Modulation) using an operating voltage of a range 4.8V-6V. This kind of motor is very common for radio control model aircraft applications, such as control of the ailerons, stabilizers and rudder. Thus, it would be very easy to buy a new servomotor if any is broken in the future.

As it will be commented in Section 2.1, the input/output interface of the robot Skybot is the Sky293 card. It performs the signal adaptation in current and voltage levels to use in different kind of devices present in robot. Its name comes from the driver motor LM293B which is included in the circuit board and it is used to amplify the signal from the microcontroller, thereby performing the motors control.

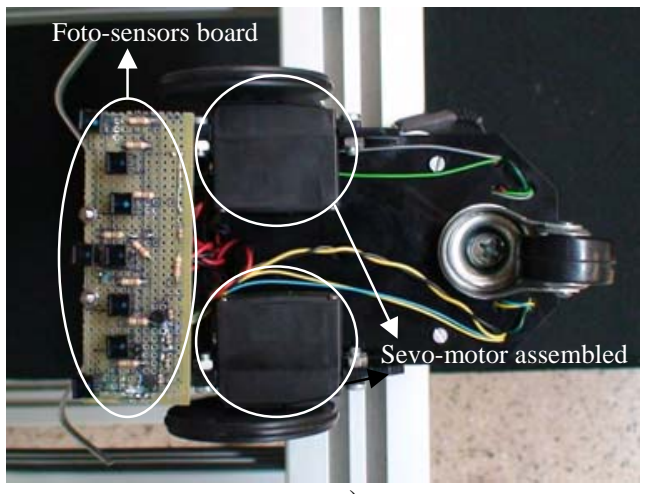

a)

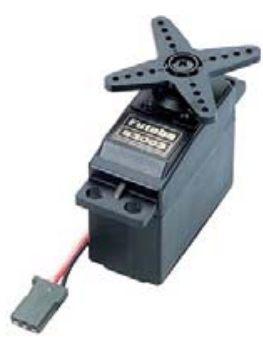

b)

Mobile robots must be moved freely in the environment. However they can only be moved 180 degrees. Therefore, a manipulation in the actuators to extract the engine and remove the caps than limit the movement of the shafts must be done. This operation is performed by students, so they transform the original servomotors in the new DC motors with 360 degrees of freedom.

The main sensors included in the Skybot robot are: four infrared sensors (CNY70), two microswitch sensors and one LDR (Light Dependent Resistor). These sensors are very low-cost and they provide 
great flexibility and versatility for the implementation of experiments by means of microcontrollers programming by students. The microswitch sensors provide a simple method to learn using digital input sensors, and LDR sensors are used to learn an analog input. The microswitch sensors are located in the front of the robot. They are used as bumpers to detect a collision. And the LDR sensor is placed in an aerial built with wire and it is used to detect light.

On the one hand, the CNY70 are placed in the front of the robot, close to the floor. Thereby, the CNY70 is used to program the robot as a line follower. The floor is white or similar and it reflects the infrared light emitted by the CNY70. Consequently, a circuit pasting black lines with adhesive tape in the floor can be designed. Thus, the infrared light emitted by CNY70 will be reflected by the white surface but not the black line. In addition, an expansion card to use eight infrared sensors instead the four original sensors was developed. This improved sensitized system allows students to develop more complex line tracker algorithms.

On the other hand, the Skybot have been extended with three LDR sensors. These sensors are used to implement algorithms to follow the light rays. In general, the different kind of sensors permits to define three types of actions to control the movement through the environment. One of the three LDR sensors is placed in the middle and the other two are placed on the sides (Fig 3a). This way, the light ray can be tracked with a sensor and other, depending on what sensor is more excited.

The main advantage of this platform is its easy extensibility. Consequently, the students can connect to the sensors network using the pin that they want, while they are connected to the microcontroller with PCB terminals with screws, which greatly simplifies the task for the students.

With regard to the movements robot, the control of the motors in the Skybot platform is carried out by means of using only four pins, two per each motor. One pin is used to select the direction and the other pin is used to turn on/off the motor. In consequence, the students' work to control the movements is simplified. Hence, it is very easy to choose an action to move among forward, backward and turn left/right. The wide versatility of Skybot allows students different ways to control and program the robot. On the contrary, other robot platforms are limited to extensions. In a normal case, a motor driver uses three pins per motor, the first pin for turn on/off and second for select the direction (forward/reverse direction). In addition, the brake is on when both pins have a high value and the motor turns freely when the both pins have a low value. Therefore, the Skybot platform is better than others for educational purpose.

\subsection{Robot programming}

Robot programming is reduced to programming its PIC16F876A microcontroller (Fig. 2). Nowadays, both assembly language and higher level languages like $\mathrm{C}$ or Pascal can be used for the programming of microcontrollers, using the appropriate compiler. From the manufacturer of the microcontroller, the student or user can find MPLAB ${ }^{\circledR}$. MPLAB ${ }^{\circledR}$ is a programming environment for PIC microcontrollers. In this environment, the student can create the program in assembler and then get the .hex file with the instructions in machine code understood by the microcontroller. The physical programming of the device would be done by a programmer, capable of recording the program contained in that hex file in the memory of the microcontroller. But MPLAB ${ }^{\circledR}$ only works under Microsoft Windows. Furthermore, for the PIC16F family, it only allows us to develop our program in assembler.

Students of this robotic course are mainly university students of computer science. Therefore they have a good knowledge of $\mathrm{C}$ programming language. It must be taken into account that each instruction written in a high level language will correspond usually with several assembly language instructions, which means that a program written in high level language takes up more memory space than one written in assembly language. However, a programmer familiar with $\mathrm{C}$ will take much less time to understand the basic structure of a program for Skybot. This is the main educational aspect regarding the programming that we want to remark in this course. Microcontroller programming using a highlevel language like $\mathrm{C}$ requires the programmer to use certain structures such as the switch in detriment of the typical if structures. Thus, students learn and understand the problem of working with limited stack memory architectures that require optimize the available resources.

One of the main reasons for the choice of microcontroller programming tools was to provide a free, multi-platform environment that allows programming the Skybot from GNU / Linux, MS-Windows, FreeBSD or Mac OS X. 
The compiler chosen was the SDCC C compiler. SDCC is a retargetable, optimizing ANSI - C compiler that targets the Microchip PIC16, PIC18 and many other microcontrollers. SDCC is Free Open Source Software, distributed under GNU General Public License (GPL). GPUTILS is a collection of tools for the Microchip (TM) PIC microcontrollers. It includes gpasm, gplink, and gplib. It's distributed under the terms of the GNU Public License. Gnu/Linux, Mac OS X, and MS-Windows are supported.

Finally, Pydownloader was chosen to download programs in the Skypic. Pydownloader is an application that uses as interface a window created with the wxPython graphical libraries. Pydownloader allows users to download .hex files previously compiled with SDCC. The Pydownloader is based on LibIris, a library programmed in Python to download programs into the Skypic card. It implements the protocol of the PIC_Bootloader. Previosly, it is necessary that the PIC_Bootloader has been recorded in the Skypic. Using this library it is very easy to implement user applications where the download programs in the Skypic is required. There are different versions of Pydownloader for both GNU / Linux, MS-Windows or Mac OS X.

The process of creating a program is very simple (Fig. 4). The students must create their C program with any text editor. Then, they compile their program with SDCC to get the hex file. This file is downloaded to the microcontroller with the Pydownloader.

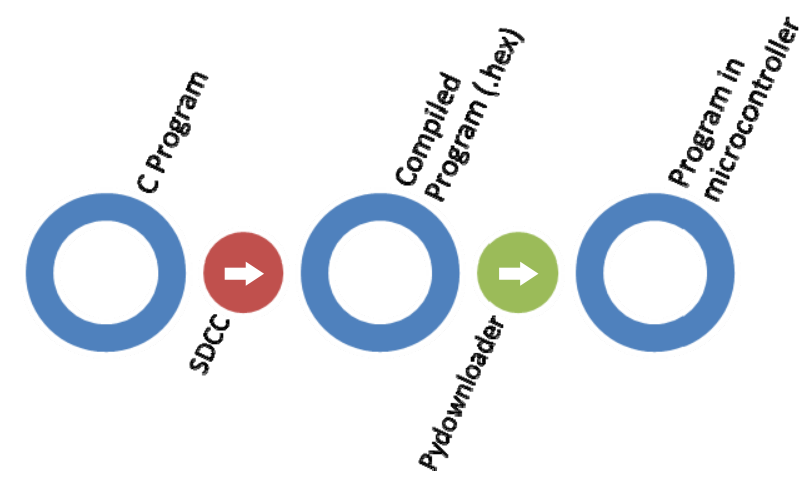

Figure 4. Scheme to download a user program for Skybot

In order to emphasize the need to create programs that take into account the hardware limitations of microcontrollers (compared with virtually unlimited resources of modern Personal Computers) it is proposed to the students perform a program based on state diagrams. Figure 5 shows the main state diagram to solve the program of the robot line-tracker. Afterwards, this program can be used in competitions as it is discussed in section 3. This figure shows that there are only three states. The robot will always be in one of 3 states:

- Always Center: When the robot is in this state, it will always choose the middle path at an intersection. To reach this state the robot has to read two lines parallel to the main line before the intersection.

- Always Right: In this state, the robot must always change to the right when entering an intersection. The main condition for reaching this stage is to find a mark on the right before the intersection.

- Always Left: The last statement may cause the robot to change direction to the left when it finds a mark on the left. While in this state, the robot should always take the left path at an intersection.

From this main diagram, the students propose the substates diagram for each of the major states that have been mentioned. Figure 6 shows the state diagram of main substate Always Right proposed by a student. All the logic of the program that solves the changing of the states of the main diagram (shown in Figure 1) is already developed into a code that is provided to students at the workshop. It operates through a voting system based on detected changes in 
sensor readings. This voting system will tell the program if it has detected a center mark (MC), a left mark (ML) or a right mark (MR).

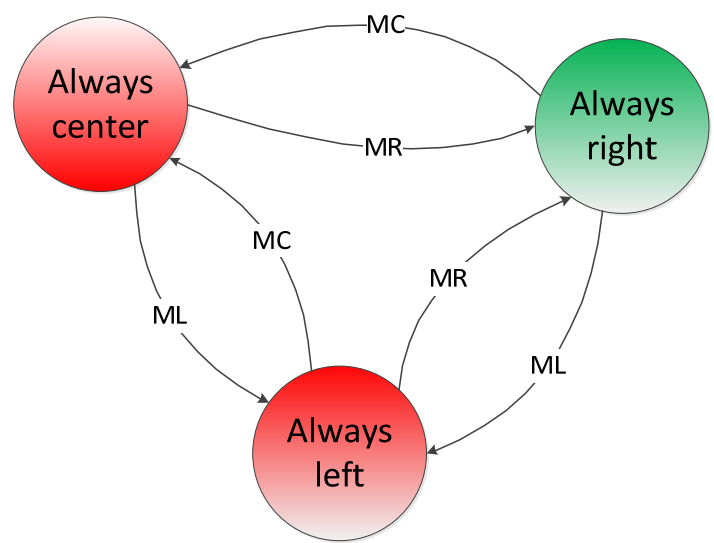

Figure 5. Main state diagram of the program for the Skybot line-tracker.

The conditions for moving from one state to another within the diagram shown in Figure 6 are defined by the reading of infrared sensors located on the front of the Skybot. Depending on the value read from these sensors, students define whether the robot should turn right, left or just keep moving forward. The " $\mathrm{X}$ " represents any sensor reading. Once the students have designed their state diagrams, it is very easy to obtain the associated program and load it to the robot for testing.

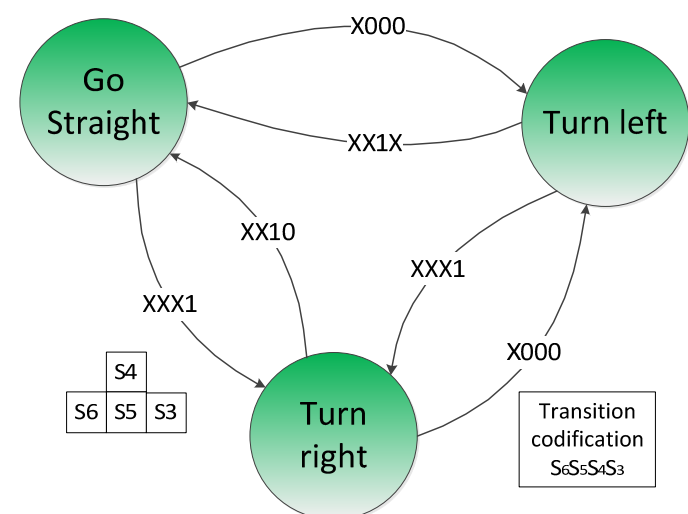

Figure 6. Substates diagram of the Always Right main state.

\section{Robotic course organization}

\subsection{Scheduled lessons and time distribution}

The authors have designed several undergraduate "Introduction to Robotics" courses, with the title "Program and build your own robot". Details about the course and the lab can be found at site of the course [28]. The first edition consisted of 32 hours divided in 15 hours of regular lecture ( $\mathrm{T}$ hours in table 2) and another 15 hours of lab (P hours in table 2) and 2 hours to do a competition among robots. The course was distributed in two week, having classes Thursday and Friday in the afternoon (between 16:00-21:00) and Saturday morning (between 9:00-14:00). The second edition consisted of 50 hours divided in 15 hours of regular lectures and 35 hours of lab. The increase in lab hours were done to take into consideration the time that the students required in the first edition to complete the robot construction. They had to dedicate after more course hours to complete the robot. The second edition had the classes distribute in 5 days, with full time dedication between 9:00-14:00 and 16:00-21:00, 
distributed in Thursday and Friday, to build the robot, and then Monday, Tuesday and Wednesday to program all the required algorithms.

The course was distributed giving the students a theoretical introduction and explanation to what they were going to perform during these sessions (Table 2). For example, if they had to build the PCB with its components, the professors explained the different components and the way of working to allow the students to understand the electronics that they are building, instead of only make the PCB without knowing what are doing.

Initially, the students had to weld the components of the sensor PCB. In addition, an extension of the original sensorial system of the robot was designed for the course. Previosly, the students had to understand the way of working of the system, this required the professor help to build the PCB.

Once they built the robot, the programming part of the course began and the teachers taught how to install the software in a computer to program the robot. Before starting to program the robot, an introduction of microcontrollers was performed, giving the students a background of how the work, their benefits and limitations.

In the programming part, the professors gave the students the indications to perform a library for the robot to read the infrared-sensors, to read the LDR and design the basic program to follow a line.

Taking into consideration the course distribution, the objectives of the course were achieved. (Qualitative data about these results is commented in section 4). The educational objectives of these courses are:

- Introduction to the robot world.

- Giving students knowledge of automatics and robotics.

- Giving students knowledge of electronics.

- Giving students knowledge of programming robots.

Table 2. Course scheduling

\begin{tabular}{cc|c|c}
\hline Activity & Hours & T/P & Number of Teachers \\
\hline Introduction and beginning with the assembly of the robot & $5 \mathrm{~h}$ & $\mathrm{~T}$ & 1 \\
Assembling the chassis of the robot & $5 \mathrm{~h}$ & $\mathrm{P}$ & 1 \\
Designing and assembling the PCB of robot & $5 \mathrm{~h}$ & $\mathrm{~T}$ & 2 \\
Assembling the PCB of the robot & $5 \mathrm{~h}$ & $\mathrm{P}$ & $\mathrm{P}$ \\
Final assembling and tuning of robot & $5 \mathrm{~h}$ & $\mathrm{P}$ & 1 \\
Installing the software & $5 \mathrm{~h}$ & $\mathrm{P}$ & 1 \\
Introduction to PIC architecture and programming Skybot & $5 \mathrm{~h}$ & $\mathrm{~T}$ & 2 \\
Examples of programming simple algorithms for PIC & $5 \mathrm{~h}$ & $\mathrm{P}$ & 3 \\
Advanced programming of the robots for the competition & $8 \mathrm{~h}$ & $\mathrm{P}$ & 3 \\
Assessment test in the competition & $2 \mathrm{~h}$ & $\mathrm{P}$ & 1 \\
\hline
\end{tabular}

\subsection{The robot competition as a motivating educational tool to assessment}

This course and the robots competition, particularly, provide an opportunity to share experiences and expertise among students. The pedagogy of teaching on robotics is still a novel field. There are a lot of motivation factors [29] including mentions as demonstration of practical and learning skills, opportunity to apply student's ideas, knowledge/experiences acquired through the contents of course, teamwork's among different engineers and/or university students with different interest in engineering, designing fun (Fig. 7b), etc.

The competition (Fig. 7a) is a good benchmark system to measure the knowledge and skills acquired in solving problems of constructing and installing robot components as well as practical knowledge in sensor measurement and behavior programming.

There are different competitions of robots like Robocup [14][30]. It gives several scenarios and variety of competitions, in an international scene. Other examples are CEABOT [16][31], it is a Spanish humanoid robot competition, and LEGO League [32] in which participle students of primary and secondary school, building the robots with standards kits.

Our competition was composed of two parts. The first part consisted in a robotracker, in which the robot must follow a circuit ofn a blank line over white background. The circuit had intersections which 
were marked in order to the robot could recognize them before arriving. Reading the mark the robot knew the turn required to perform the shortest way to the goal in that intersection. A mark in the left side meant that the shortest way was to turn left in the intersection. A mark in the right side meant that the shortest way was to turn right in the intersection. A mark in both sides of the main line meant that the shortest way was the line of the middle in the next intersection. This circuit allowed teachers to determine the best student's program for robot. This program had the best intersection detector and it permited to follow the line in the best way possible.

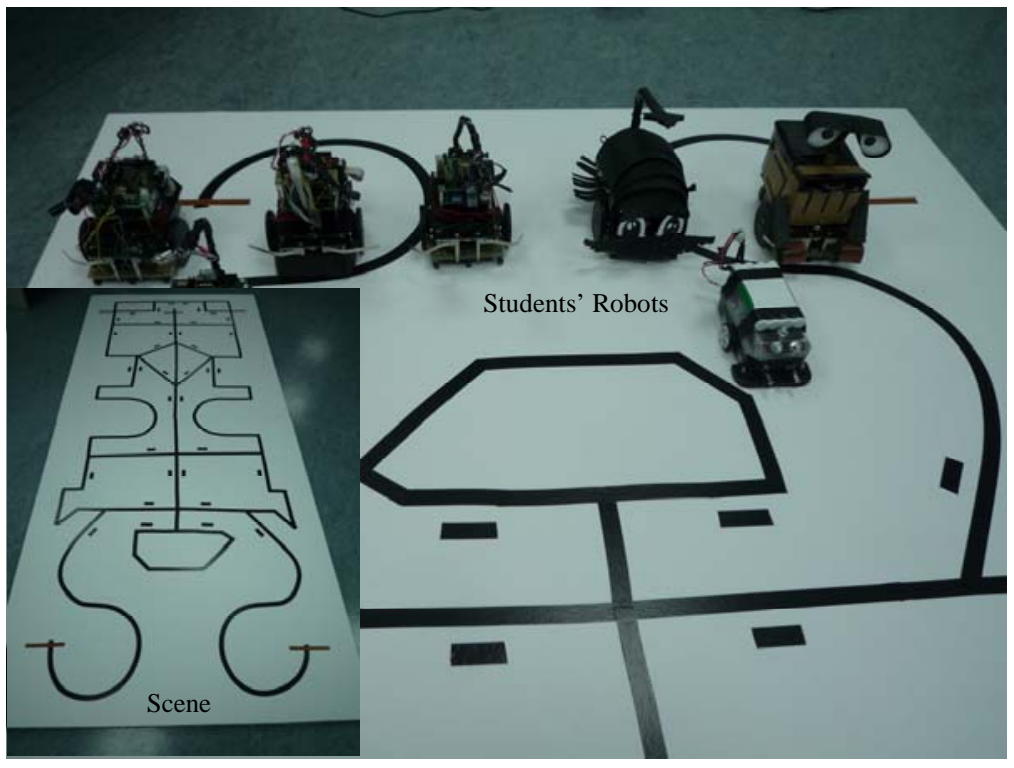

a)

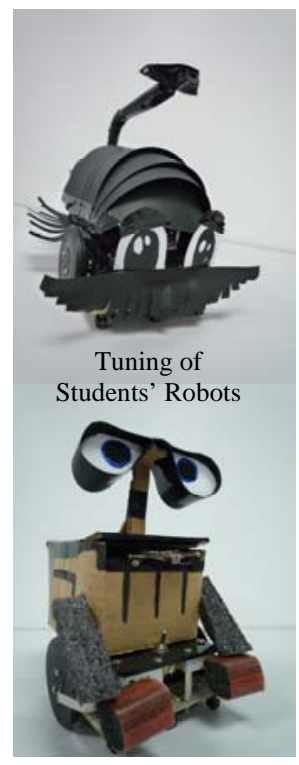

b)

Figure 7. a) Competition venues. b) Students’ robots

The second part of the competition consisted in a balloon fight. In this case, for each robot a small balloon and a high intensity led were attached. In this part of the competition the robots had to detect the led of the other robot and using that information to move the other robot away of the competition area of explodes. This part of the competitions allow the students to know the performance of the robots and the difference of using a theoretical approach a real approach in the programming, because the ambient conditions are not always the same. Some videos of the competitions can be viewed in [28].

\section{Student's opinion about the course}

During the last year 2011, second edition, the course about building and programming of robots were taught to undergraduate students with different university studies. Generally, they achieved the engineer's degree from Computer Science, Telecommunication and Industrial engineering. The number of students was 20 and, they gave volunteer opinions, from a survey designed by the teachers. Sixteen important questions were consulted. The scale of qualification was between 5 (totally agree and/or satisfied) and 1 (totally disagree and/or dissatisfied) for the survey.

Three questions were about what were the knowledge levels learned from interdisciplinary sources such as electronic, robotics and programming. The result of this opinion is shown in the Fig. 8 . Analyzing the results in detail:

- $\quad$ Electronic: The $40 \%$ voted 5 and $60 \%$ have voted 4 in a scale between 1 and 5 . In general, the students think that the knowledge level learned is very satisfactory compared to expectations that they had before starting. 
- $\quad$ Robotics (Control and Computer Science): The 45\% voted 5 and 50\% voted 4 . The students think that the broad range of robotics topics such as effectors/actuators, manipulation, control architectures, sensors and locomotion/navigation were successfully learned.

Programming: The students implemented and tested their algorithms on the Skybot platform. They programmed the device drivers and behavior robotics and these codes can be ported directly on the physical robot platform. In relation to these aspects, the students' opinion was more diverse but always in a medium-high level of satisfaction. The $40 \%$ voted 3 (medium satisfied) compared to $25 \%$ and $35 \%$ voted 4 or more (high and very high satisfied).

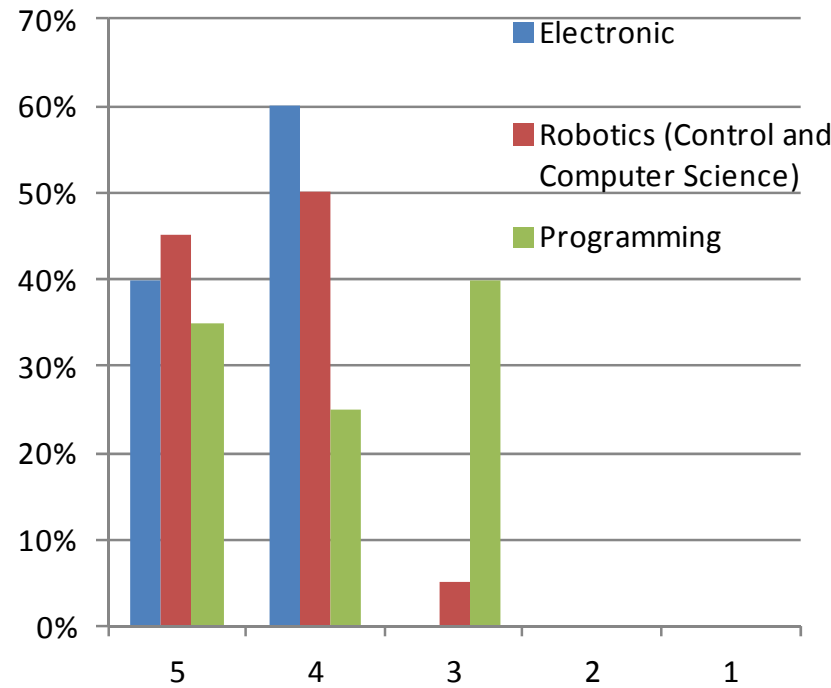

Figure 8. Self-assessments of knowledge acquired in the three interdisciplinary areas commented.

Moreover, the students were asked if the course model was well-defined in relation to structure and contents, course duration and attendance and, overcoming of difficulty course. In these questions, the teachers wanted to know if the students considered the organization of robotic course appropriated to facilitate learning process. The opinion survey consisted of 4 additional questions about:

- $\quad$ Learning difficulty level: Effort level required to learn according to the previous knowledge and concepts given in the course

- $\quad$ Duration: Number of hours that students spend studying and experimenting for each hour in the classroom. These hours are used to develop the activities and experiments planned by teachers.

- Documentation: Quality of educational resources such as PPT, PDF files, websites, educational videos, etc. The survey attempted to find answers to these questions. Did they the learning more fun? Were they explanatory and illustrative? Were they suitable to learn to students with different interdisciplinary training?

- $\quad$ Structure and contents: Quality of planning of the seminars in the classroom. Were they suitable to achieve the objective proposed in accordance with the provided documentation by the teachers and with the time to do the activities and experiments?

A comparison of these four aspects demonstrates the acceptance level of the course for the learning processes in a robotics class (Fig. 9).

The students consider (they voted 3 in the great majority) that the course is neither easy nor difficult. Some students commented that there were too many hours of programming tasks of device, microprocessors and/or sensors. However other many students think that these are insufficient and they have to spend even more time. In relation to the global duration of course, the $35 \%$ of students voted 4 
or more points (they agree with the hour numbers spent and they consider adequate and sufficient the course duration). Only 30\% of students voted that more hours are required for training. The structure and contents have been well worth (60\% voted 4 or more, so they were satisfied or very satisfied) and the rest of students not gave their opinion. Something similar happened when the documents offered by teachers are valued. Thereby, the $40 \%$ of students were satisfied o very satisfied with them and the rest was silent (Don't know/Not available).

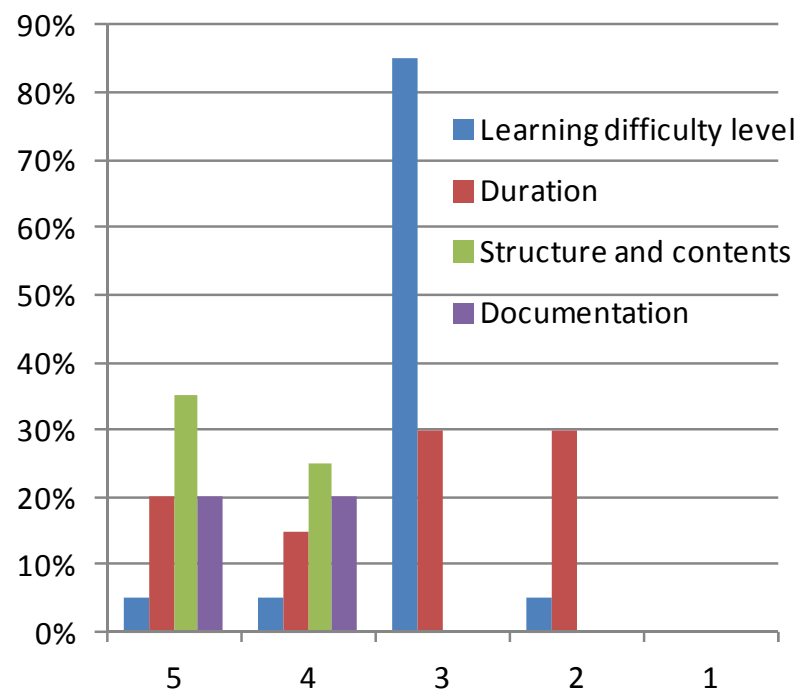

Figure 9. Students' opinion about the organization of robotic course.

The course covers real-time programming techniques, microcontroller interfacing and minirobot navigation/behavior by sensors. To do this, the students worked to construct their own robot with Skybot materials [17]. Afterwards, the students could specify the tasks to be carried out by their robots thanks to the knowledge acquired with the methodology of course.

In recent years, the design of minirobots has been introduced for teaching at different levels, also at the Universities. There are a lot of robot platforms to teach robotics. The most popular architectures are based on wheeled platforms with an onboard control unit. Skybot is an example. What offers Skybot in relation to electronic, microcontrollers (assembly/high-level language), and minirobotics (kinematics, dynamics, sensors, control theory)? The survey has tried to give answer to this question. So, the results of survey show that, according of the student opinions, the Skybot can be useful as robotic kit to teach and learn robotics and their interdisciplinary skills (Fig. 10). Thereby, the students opined that the knowledge and skills in electronics were extensively covered using Skybot $70 \%$ of students were strongly agree and $25 \%$ were agree). Similarly, the $85 \%$ of students thought that Skybot satisfied the need for learning robotics (60\% of students were strongly agree, $25 \%$ were agree against $15 \%$ of students were silent). Furthermore, the $95 \%$ of students voted 4 or more when they were asked about the use of Skybot and Arduino to learn Microcontrollers.

The preferences about employers of engineering industries were based on to recruit future engineers with two important skills. They have to be autonomous and contribute with own and innovate ideas independently. And, they have to be able to work in group when it is required. In general, the engineering industries want new graduated who be able to 'know-how' to solve real-world problems.

This robotics course incorporates activities as robotic competition. This activity improves the learner's sense of development of personal skills with feedback from the teacher, the study program and the ideas presented from other students and peers.

On the one hand, the students developed an autonomous learning when they implemented their own algorithms for the competition. This activity implies responsibility for one's own learning behavior.

On the other hand, this type of activity was designed to satisfy the need for relatedness through games and competitions-playing. In addition, the competition provides extra motivation that helps to develop new ideas and to obtain better results. However, measuring a person's skills and ability to 
apply acquired knowledge is subjective and very difficult. Some questions of the survey were to assess the perception and the expectation of the students on employability skills for a future job (Fig. 11a). Furthermore, the students were asked about the possibility to study other new courses about robotic (Fig. 11b). In our opinion, it is necessary that students get some background knowledge wider than that achieved with this basic course. For this reason, the students are encouraged to continue the learning in robotics. To measure this motivation, the students were interviewed about if they were interested in new robotics course to further increase their knowledge and skills in new situations, especially in fields as engineering and robotics.

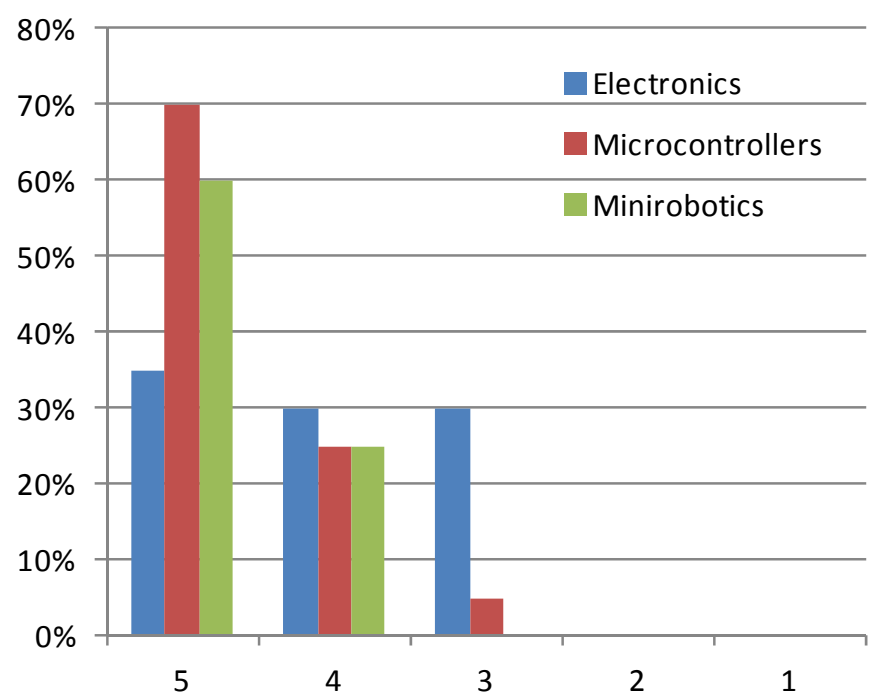

Figure 10. Students’ opinion about Skybot platform to learn robotics.

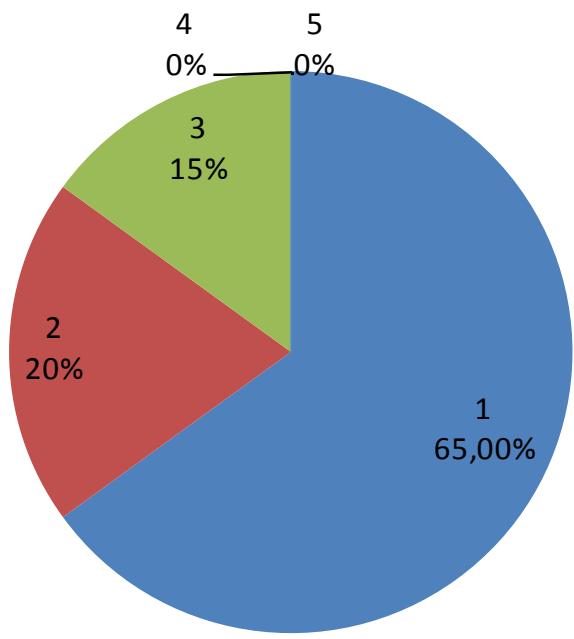

a)

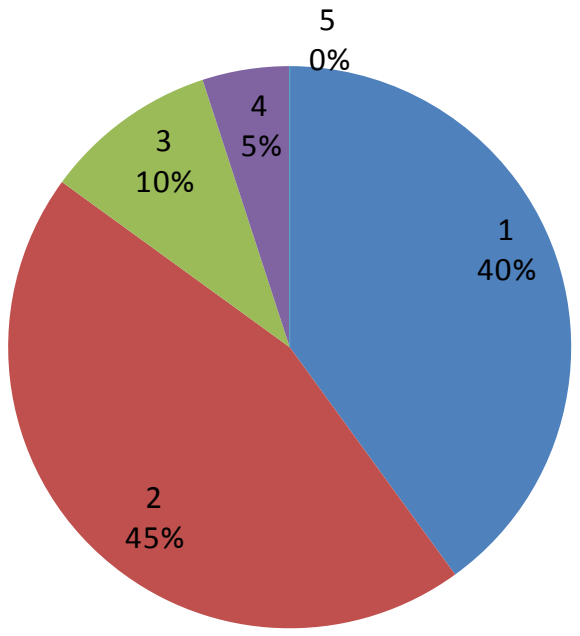

b)

Figure 11. Students' opinion about development of personal skills to professional future: a) Robotics course is useful to professional future? b) Are you interested in new advanced robotics course?

\section{Conclusions}

This paper shows a robotic course with a teaching methodology based on practical classes that harnesses basic electronic components to build a low cost educational robot. The building 
of robots enables students to learn concept relationships such as electronic, control and automation. This paper has shown the design and development of lessons about electronic and control concepts in an integral robotic course. The design of this course includes e-learning materials, manuals, basic electronic hardware, free-open software for programming, etc. Each student develops its own robot, no two identical robots. This way, the course serves to encourage to the students. Furthermore, they can power its skills in robotics and to verify the results and the learning acquired from experiments. So, for the evaluation of this course in terms of the accuracy of learning concepts, the students participated in a robot competition which tested the student's developments with the robots built themselves. The competition is a motivational aspect for the students. The reason is the relevance of this type of competitions has on the today's society. In this case, this course and its competition has had a particular impact in the local, regional and national press and television, the published information can be followed through some links in the site http://www.aurova.ua.es/microbot/index.php/es/prensa. Moreover, teachers have evaluated the student satisfaction and the opinion of this robotic course by means of an anonymous questionnaire.

\section{Acknowledgment}

This work is supported by the "Education Science Institute" and the "Technology \& Educational Innovation Vice-President Office" of the University of Alicante through the aid "Technologic \& Educative Research Groups”.

\section{References}

[1] F. B. Vavassori, "Exploring the educational potential of robotics in schools: A systematic review", Computers \& Education, vol. 58, no. 3, pp. 978-988, 2012.

[2] NAO robot web site: http://www.aldebaran-robotics.com/

[3] M. S. Couceiro, C. Figueiredo, J. M. A Luz, N. M. F. Ferreira, R. P. Rocha, “A Low-Cost Educational Platform for Swarm Robotics”, International Journal of Robots, Education and Art, vol. 2, no. 1, 2012.

[4] Vex web site: http://www.vexrobotics.com/

[5] K-Team Mobile Robotics web site: http://www.k-team.com/

[6] Lego Mindstorms web site: http://mindstorms.lego.com/

[7] I. R. Nourbakhsh, K. Crowley, A. Bhave, E. Hamner, T. Hsiu, A. Perez-Bergquist, S. Richards, K. Wilkinson, "The Robotic Autonomy Mobile Robotics Course: Robot Design, Curriculum Design and Educational Assessment”, Autonomous Robots, vol. 18, pp. 103-127, 2005.

[8] F. Klassner, S. D. Anderson, "LEGO MindStorms: Not Just for K-12 Anymore”, IEEE Robotics and Automation Magazine, vol. 10, no. 2, pp. 12-18, 2003.

[9] J. C. Cañada, T. J. M. Sanguino, S. Alcocer, A. Borrego, A. Isidro, A. Palanco, J. M. Rodriguez, "From Classroom to Mobile Robots Competition Arena: An Experience on Artificial Intelligence Teaching”, International Journal of Engineering Education, vol. 24, no. 4, pp. 1-8, 2011.

[10]D. Assaf, R. Pfeifer, "EmbedIT - An Open Robotic Kit for Education”, In Proceedings of Research and Education in Robotics - EUROBOT 2011, pp. 29-39, 2011.

[11] R. R. Murphy, “Competing for a robotics competition”, IEEE Robotics \& Automation Magazine, vol. 8, no. 2, pp. 44-55, 2001.

[12]R. Balogh, “A Survey of Robotic Competitions”, International Journal of Advanced Robotic Systems, pp. 144-160, 2008.

[13] T. Balch, H. A. Yanco, "Ten years of the AAAI mobile robot competition and exhibition: looking back and to the future”, AI Magazine, vol. 23, no. 1, pp. 13-22, 2002.

[14]H. Kitano, M. Asada, I. Noda, H. Matsubara, "RoboCup: Robot World Cup”, IEEE Robotics and Automation Magazine, vol. 5, no. 3, pp. 30-36, 1998.

[15] M. Hernando, R. Galan, I. Navarro, D. Rodriguez-Losada, "Ten Years of Cybertech: The Educational Benefits of Bullfighting Robotics”, vol. 54, no. 4, pp. 569-575. 
[16] M. Gonzalez-Fierro, A. Jardon, S. Martinez de la Casa, M. F. Stoelen, J. G. Victores, C. Balaguer, "Educational initiaves related with the CEABOT contest", In Proceedings of the International Conference on Simulation, Modeling and Programming for Autonomous Robots, pp. 649-658, 2010.

[17]F. Torres, F.A. Candelas, S.T. Puente, J. Pomares, P. Gil, F. Ortiz, "Experiences with Virtual Environment and Remote Laboratory for Teaching and Learning Robotics at University of Alicante”, International Journal of Engineering Education, vol 22, no. 4, pp. 766-776, 2006.

[18] C.A. Jara, F.A. Candelas, P. Gil, F. Torres, F. Esquembre, S. Dormido, "EJS+EjsRL: an interactive tool for Robotics simulation, Computer Vision processing and remote operation. Application to virtual and remote laboratories”, Robotics and Autonomous System, vol. 59, no. 6, pp. 389-401, 2011.

[19] C.A. Jara, F.A. Candelas, S.T. Puente, F. Torres, "Hands-on Experiences of undergraduate students in automatics and robotics using a virtual and remote laboratory", Computer \& Education, vol. 57, no. 4, pp. 2451-2461, 2011.

[20] Skybot robot. Online: http://www.iearobotics.com/wiki/index.php?title=Skybot

[21] I.W. Park, J.O. Kim, "Philosophy and Strategy of Minimalism-based User Created Robots (UCRs) for Educational Robotics - Education, Technology and Business Viewpoint”, International Journal of Robots, Education and Art, vol. 1, no. 1, pp. 26-38, 2011.

[22] A. Prieto. Wiki of the SKYPIC training board for PIC microcontrollers. Online: http://www.iearobotics.com/

[23] Microchip web site: http://www.microchip.com/

[24] Arduino web site: http://www.arduino.cc/

[25] Netduino web site: http://netduino.com/

[26]SparkFun Electronics. IOIO for Android Beginners Guide. Online: http://www.sparkfun.com/tutorials/280

[27] RaspBerry web site: http://www.raspberrypi.org/

[28] Introduction to Robotics: Program and build your own robot. Online: http://www.aurova.ua.es/microbot/index.php/es/segundaedicion/segundaedicion

[29]D.J. Pack, R. Avanzato, D.J. Ahlgren, I. M. Verner, "Fire-Fighting Mobile Robotics and Interdisciplinary Design-Comparative Perspectives”, IEEE Transactions on Education, vol. 47, no. 1, pp. 369-376, 2004.

[30] Robocup web site: http://www.robocup.org

[31] CEABOT web site. Online: http://www.ceautomatica.es/og/robotica/concurso-ceabot

[32] LEGO league web site: http://www.firstlegoleague.org/ 